\title{
Design on curves of wall-thickness for Blow Molding Machine based on HMI
}

\author{
Cheng Yayun ${ }^{1, a}$, Zhu Zhisong $^{1, a}$ and Liu Jing ${ }^{1}$ \\ 1 School of Mechanical Engineering,Nantong University, NanTong, JiangSu, China
}

\begin{abstract}
Properly control of blow molding machine's wall-thickness relates to the quality of plastic products, design on curves is the key technology of blow molding machine's wall-thickness control.Adopted cubic spline curve as a fitting prototype, used advanced HMI as a design platform, by cooperation with HMI configuration function and macro code,an interface of curves was designed,a good performance of human-computer interaction and core data's transmission in the whole machine were realized.By using this method,the integration of the system was increased and costs was reduced dramatically.
\end{abstract}

\section{INTRODUCTION}

Recently, blow molding has become one of the most widely used methods in the production of plastics. Plastic products specifies the thickness according to its intensity, but when in the stage of parison formation, extrusion swell, drop and rebound,such changes do often occur, which will lead to the products' uneven thickness. So control for the parison wall-thickness is a key technology to ensure the quality, improve production efficiency and save materials[1-3]. In our country, a imported and independent wall thickness control system was often putted, with the mechanical part of the blow molding machine added into a blow molding processing equipment. But do this, the cost will be increased, because such a complete machine is always not cheap.

Control for the parison wall-thickness can be divided into axial and radial control. To realize axial control, make mandrel do axial movement along the preset position to change die clearance[4]. Users can touch the HMI to set the control points, do interpolation, fit out cubic spline curve, discrete coordinate points out and then transmit to the whole machine. Later on, a closed loop was adopted in the control, variable intensity current or voltage signals were outputted by curves, then die clearance driven by proportional servo drives mandrel between inner and outer molds was adjusted, the outflow of the raw materials was controlled, finally the goal of adjust the axial wall-thickness dynamically was realized . In this design we mainly focus on the research of axial wall-thickness, aim at the most core part--curves of wallthickness. A design idea which taking HMI as a curve design platform was putted forward and cubic spline curve as a fitting prototype was adopted.

\footnotetext{
a Corresponding author: zhu.zhs@ntu.edu.cn
}

\section{Requirements of interface design}

To operate HMI conveniently, some necessary functions should be set in the interface of curves:

(1)Click the reset button and clear the plotting area before plotting the curve.

(2)Get some control points in the area and click the calculate button, the system start calculation when plotting the curve; Click the draw button, plotting the curve automatically; Discrete the curve and then transmit coordinate points to the control system.

(3)Click the draw area after plotting the curve, no changes occur.

\section{Choose of curve}

Design principles of curve: to ensure the quality of the parison's surface, every wall-thickness point to reach the settled goal is required. Also the transition between the wall thickness can not too big, it shall be as smooth as possible.

In order to fit out a smooth curve through the settled points, numbers of points like $\mathrm{Xi}(\mathrm{I}=1,2, \ldots, \mathrm{n})$ are given. Though the common method to interpolate can guarantee the continuity of each point, but can also bring poor smoothness; High polynomial interpolation is easily to arose Runge phenomenon, bring lots of problems like large amounts of calculation, curve deformation[5]. Classic Bessel curve interpolation can ensure that all points be of continuous and differentiated, but if alter somewhere of the curve, overall could changes[6], which would undermine the original wall thickness, greatly reduce the efficiency of design. A piece-wise interpolation polynomial is used to approximate curve 
will bring excellent effect for its simple and adaptation. Cubic spline interpolation belongs to the small deflection curve, which can fitting accuracy and meet the requirements of wall-thickness curve. In consideration of curve's performance and computational complexity, the method of cubic spline interpolation to fit the curve is adopted.

\section{Design of system hardware and software}

HMI has a set of transparent absolute coordinate location system with the most convenient and natural way of human-computer interaction. The system generally includes two parts, one is a HMI controller, another is a HMI detection device. The controller receives the information from the detection device, and then converts it to coordinates while touched by an operator's finger. Previous researchers just takes advantage of the screen's display and $\mathrm{I} / \mathrm{O}$ function, while curves of wall-thickness were designed by an independent wall-thickness control hardware[7]. They ignored that its macro code can perform fitting operation besides human-computer interaction, which can reduce hardware costs[8].

\section{1 the design of system hardware}

Kinco company's MT4620TE is applied in this design, it's a four-wire resistive analog touch screen, and its development software is a configuration software called EV5000 based on PC. PLC is adopted to control the whole machine, tie that connected PLC and HMI is a serial port.

The main works are as follows:

(1)Collecting touch points' coordinates;

(2)Operating and fitting the curve;

(3)Drawing curves of wall-thickness.

\section{2 the design of system software}

System software is designed according to this idea: the script is written for the cubic spline curve in the configuration software, $\mathrm{X}-\mathrm{Y}$ element is used to show the curve.

When HMI is touched, relevant event will be set of, the script will be activated, eventually the draw of the curves of wall-thickness finished.

The entire flow is illustrated in Figure 1.

\section{3 the design of interface}

Figure 2 shows interface of HMI, figure 3 shows the basic idea of interface design, plotted by material storage cylinder's displacement on the horizontal axis and discharge port cylinder's on the vertical. Get two buttons correspond to each macro code area.

Register data are cleared by the reset button, interpolation on coordinates are done by the draw button, curve's 100 segments are discrete by piece-wise function, 101 wall-thickness points are transmitted to the PLC.
After optimization, HMI draws the curve of wallthickness.

\section{Realization process}

Macro code use the standard C scripting language, working closely with relevant components, can realize logical functions[9]:After debug, save, compile in PC, conduct offline simulation to confirm the normal operation, then download to HMI through configure the network port.

\section{1 establishment of coordinate axis}

Considering HMI is a absolute coordinate location system, and its origin is at the upper-left corner, this is different from most users' operating habits.

So the coordinate system is moved by a circumvolving and shifting operation, then the origin is moved to the lower-left corner, finally the transformation of the physical coordinates to logical ones is realized.

Figure 4 shows that area "OBCD" represents HMI's panel, "O'B' $C$ ' $D$ "” represents drawing area, origin " $O$ " is based on physical coordinate, " $\mathrm{O}$ "' is a specified origin in drawing area.

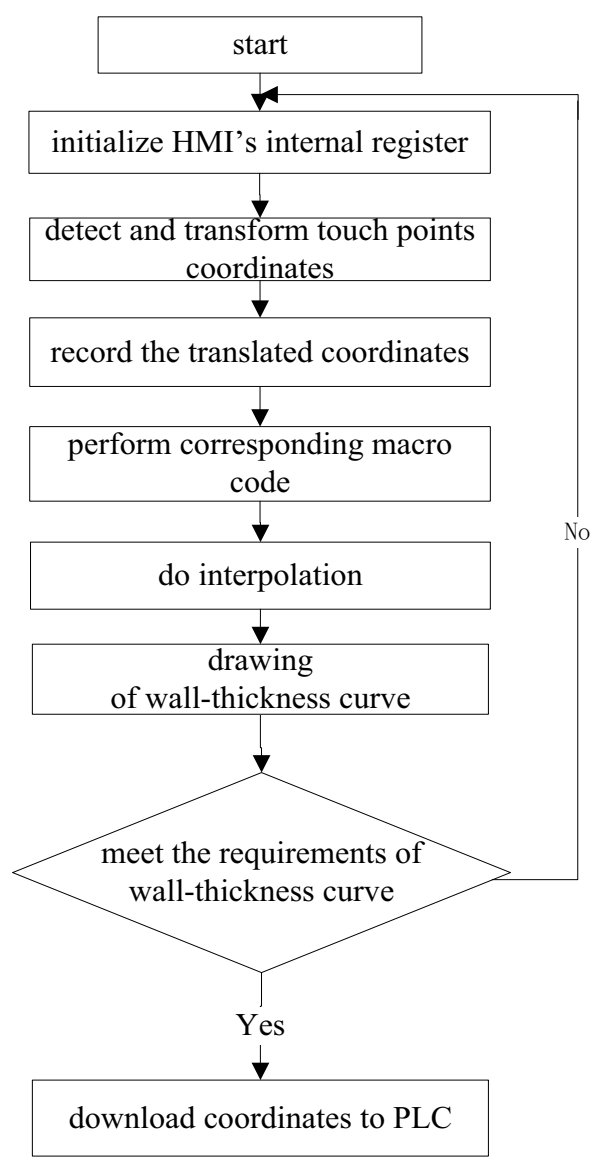

Fig 1. Process of Software Design 


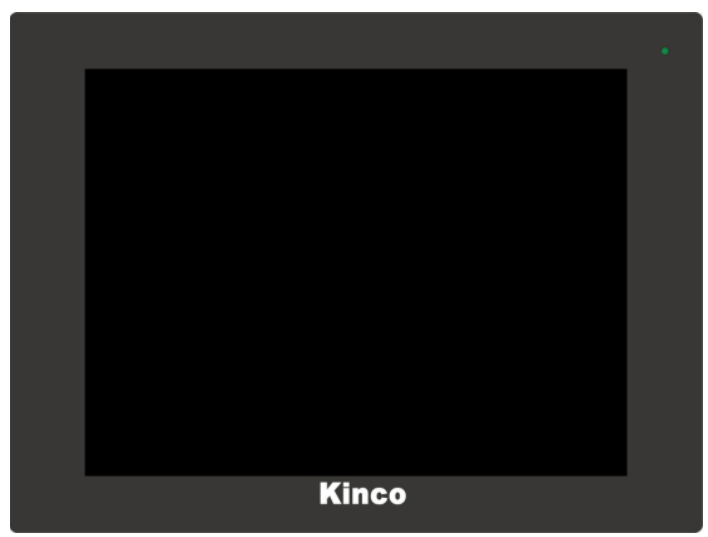

Fig 2. Interface of HMI

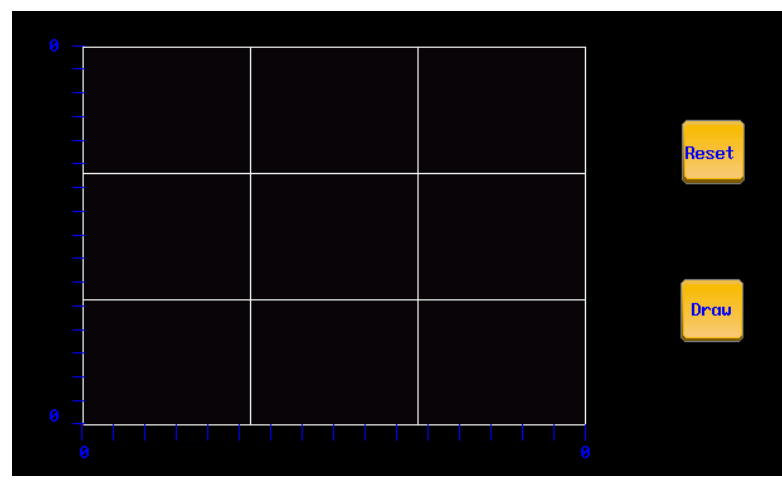

Fig 3. Design of Interface

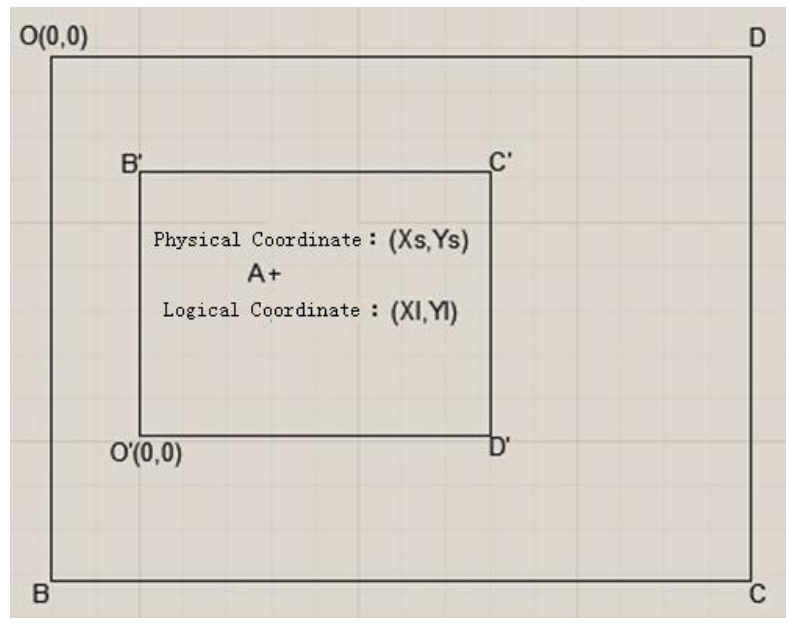

Fig 4. The Transformation of Coordinates

\section{2 code design}

C scripting language of cubic spline curve is written in this step, transmitted to HMI macro code area[10]. Contents including define internal and external variables, assign values to input variables, solve equations of cubic spline curve, output control coordinates, draw the curve, eventually save and compile macro code.Figure 5 shows HMI.

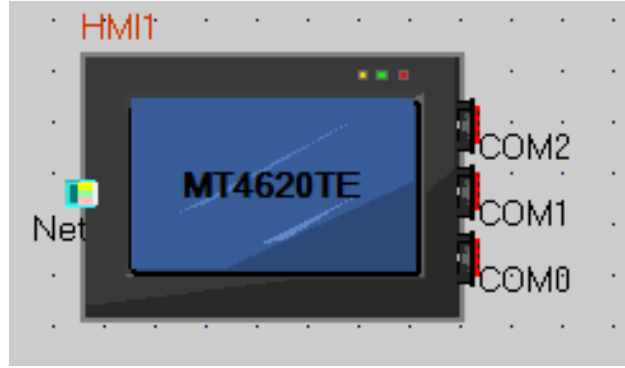

Fig 5. HMI

Table 1 lists the main variables used in the design of the code.

\begin{tabular}{|l|l|l|l|l|}
\hline $\begin{array}{l}\text { Data } \\
\text { Type }\end{array}$ & $\begin{array}{l}\text { Variable } \\
\text { Name }\end{array}$ & $\begin{array}{l}\text { Address } \\
\text { Type }\end{array}$ & Address & Length \\
\hline bit & LB100 & LB & 100 & none \\
\hline $\begin{array}{l}\text { unsigned } \\
\text { short }\end{array}$ & LW1 & LW & 1 & none \\
\hline $\begin{array}{l}\text { unsigned } \\
\text { short }\end{array}$ & aa & LW & 100 & 101 \\
\hline $\begin{array}{l}\text { unsigned } \\
\text { short }\end{array}$ & bb & LW & 300 & 101 \\
\hline $\begin{array}{l}\text { unsigned } \\
\text { short }\end{array}$ & X & LW & 500 & 202 \\
\hline $\begin{array}{l}\text { Data } \\
\text { Type }\end{array}$ & Function \\
\hline $\begin{array}{l}\text { bit } \\
\text { two stages of 0 and 1,trigger the operation of } \\
\text { macro code }\end{array}$ & $\begin{array}{l}\text { record the number of touch points,be } \\
\text { convenient to call }\end{array}$ \\
\hline $\begin{array}{l}\text { unsigned } \\
\text { short }\end{array}$ & $\begin{array}{l}\text { record touch points' horizontal coordinates } \\
\text { unsigned } \\
\text { short }\end{array}$ & \multicolumn{2}{|l|}{} \\
\hline $\begin{array}{l}\text { unsigned } \\
\text { short }\end{array}$ & record touch points' vertical coordinates \\
\hline $\begin{array}{l}\text { unsigned } \\
\text { short }\end{array}$ & $\begin{array}{l}\text { record touch points' horizontal and vertical } \\
\text { coordinates }\end{array}$ \\
\hline
\end{tabular}

LB100 is a state switching register, it has 0 and 1 two states, involved in the three macro code areas.LW1 represents the number of touch points, its macro code variables should be set in both the input and operate macro code areas. Because such variable has a function of write in the input code area, while in the operate code area, it has a function of read, so it is defined as "read/write."

\subsection{1 input code design}

"key button" element is used to set a touch area under the configuration edit interface--"XY graph" element, finally to achieve the following two functions: count and call macro code. As it shown in figure 6, internal register 9030 and 9031 are used to collect touch points' coordinates, then address is divided,the number of touch points are stored in register LW1, horizontal and vertical coordinates are deposited separately in register LW100 and LW300, which is convenient to call. 


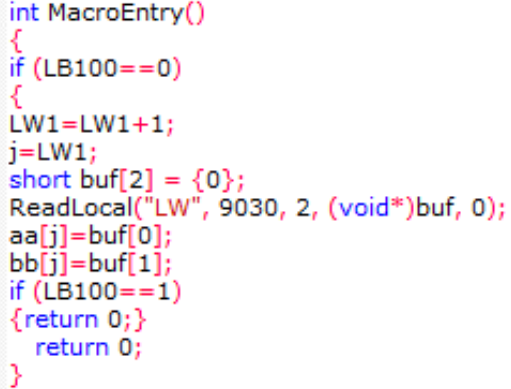

Fig 6. Key Code No.1

\subsection{2 operate code design}

This $\mathrm{C}$ scripting language is aimed at fitting cubic spline curve. The transfer of the internal data relies on the register, and the connection of two macro codes is maintained by the same register, so coodinate variables which record wall-thickness control points should be defined in the window of variables, and the information can't be changed.

"XY graph"element is used to intuitively reflect the relationship between two variables, then a sequence of continuous registers'data is read by the cycle time, and mapped in a form of biaxial curve. As it shows in figure 7, after mapped, 101 pairs of interpolation points' horizontal and vertical coordinates are stored separately in two array variables, then all these coordinates are stored in a form of registers with the beginning of LW500 that addresses are continous, ranked from X1, Y1, X2, Y2 to X100, Y100, X101 and Y101.

Attention:In order to call, the final array variable cannot be defined in the code edit ar, it can only be defined in macro code variables window.

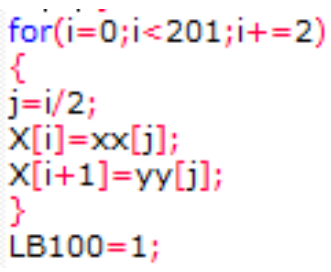

Fig 7. Key Code No.2

\subsection{3 reset code design}

We need perform a call function similar to the $\mathrm{C}$ language, while it doesn't support to call each other between macro and macro, so it can be only set a flag bit to trigger the operation of other macros in the code .

As it shown in figure 8, when $\mathrm{LB} 100=0$, "input" macro code is triggered, click event is accepted by touch area and the corresponding points' coordinates are recorded. After collecting, click "draw" button,perform "operation" macro code, meanwhile set LB100 to 1, the curve generated and showed. The touch area is not available at this time, so regardless arbitrary click of the area, the curve will not change. When "reset" button is pressed, LB100 is set to 0 , all the internal registers are reset, draw area are cleared, another design is ready for starting.

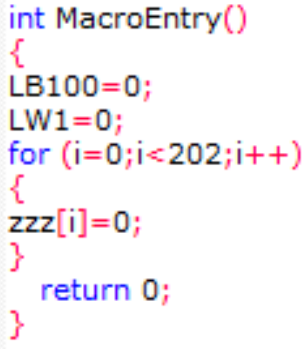

Fig 8. Key Code No.3

\section{3 drawing effects}

Just like Fig.9 shows, a smooth and continuous curve can be plotted when HMI is touched. All points and curves can be cleaned up by selecting reset button. The maximum displacement of material storage cylinder is $400 \mathrm{~mm}$, and discharge port cylinder' is $300 \mathrm{~mm}$, the two values can be changed according to the actual demand at any time.

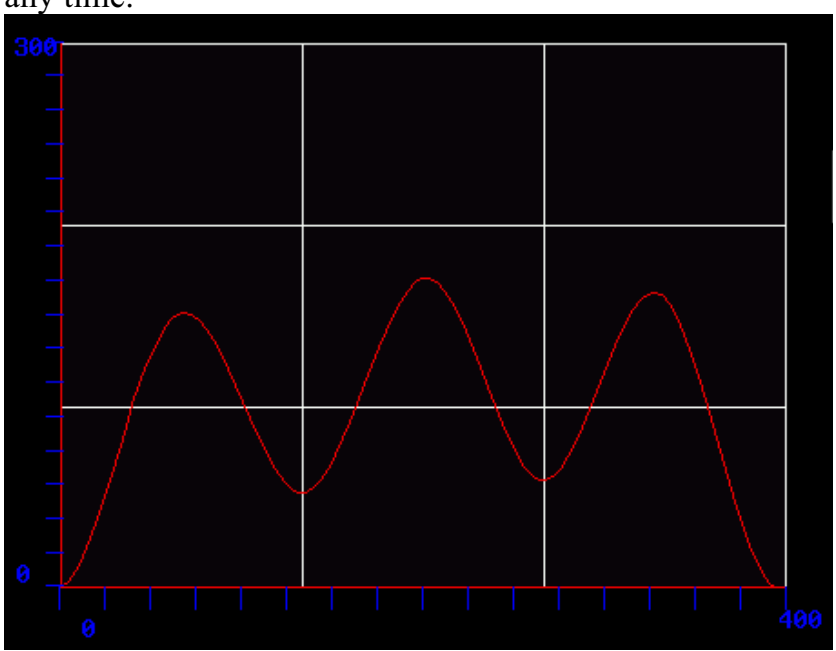

Fig 9. Effect of the Drawing

\section{Conclusion}

Select HMI as a curve design platform can reflects its good interactivity and can intuitively reflects the change of the wall-thickness data,improves the integration of the system, reduces the complexity of the wall-thickness control system, better helps reducing the cost of hardware. It no longer requires professional and technical staff to alter the program, but just asks operator to click some control points and then fit a curve according to actual demands, which is able to adapt the high efficiency of the actual products and change the complicate situation. On this basis, combining with the specific requirements of blow molding machine's system, a low cost and good performance wall-thickness design plan can easily be developed.

\section{Acknowledgments}

I would like to express my gratitude to all those who helped me during the writing of this paper. 
My deepest gratitude goes first and foremost to Professor Zhu zhisong,my supervisor, for his constant encouragement and guidance. He has walked me through all the stages of the writing. Without his consistent and illuminating instruction, this paper could not have reached its present form.

Last my thankful to all those who helped me, like sister Liu, who gave me a lot of help.

\section{References}

1. X.F.Xu, Y.X.Gao: Mechanical Engineering and Automation,

2. 2013,(2):141-142

3. J.S. Zeng, X.F. Zeng:China Plastics Industry, 2010, 38(9): 49-52

4. Gengqun Huang, Hanxiong Huang: Journal of Materials Processing Technology, 2007,182(1-3): $512-518$

5. Y.G. Zhang: Hydraulics Pneumatics and Seals, 2011(7): 1-4

6. Q.Zhu: Journal of Hunan University of Science and Engineering,2005,26(11):206-208

7. Z.Y.Zhang: SiChuan University of Science and Technology,

8. 1998,17(4):32-35

9. L.H. Zhang, P. Zhang, J. C Qiu, etal: Plastics Science and Technology,2013,42(4):115-119

10. Information on http://www.kinco.cn/ 